\title{
Improved Monitoring and Surveillance through Integration of Artificial Intelligence and Information Management Systems
}

\author{
Marco Lazzari Paolo Salvaneschi \\ ISMES - viale Giulio Cesare, 29 - 24124 Bergamo ITALY
}

Fax: +3935211191 Tel: +3935307333

\begin{abstract}
The paper describes the results of a project which aims to improve the capabilities of an information system (IS) which supports the management of safety of dams. The improvement has been achieved through the incorporation of additional components developed using artificial intelligence concepts and technologies.

We describe the pre-existing IS (comprised of automatic monitoring systems, telemetry and data bases), identify user requirements driving the evolution of the IS and explain how AI concepts and technologies may contribute. We describe the functions, the architecture and the AI techniques of two systems (MISTRAL and DAMSAFE) added to the IS. Moreover we discuss the issue of integration of the AI components and the pre-existing system and we present the technology developed to support this process.

Finally we give the implementation status of the project (which delivered components operational since 1992) and some information about the user acceptance, development effort and applicability to other fields.
\end{abstract}

\section{0 . The context}

This paper derives from a project which aims to improve the capabilities of an existing information system supporting the management of safety of dams. The improvement has been achieved through the incorporation of additional components developed using AI concepts and technologies. Significant resources are expended in the field of civil engineering in managing the safety of large structures like dams. In Italy, the collection, storage and analysis of information concerning a dam is considered as a critical part of managing safety.

Automatic instrumentation and data acquisition systems are used to monitor the real time behaviour of dams. The output of monitoring systems is presented locally to dam wardens to alert them to possibly dangerous situations. Telemetry systems are used to send the information to a central data base where experts evaluate the status of the structure through the interpretation of data.

The difficulty associated with this data interpretation (both at local and at central level) is due to different factors. Among them: the large amount of data; the uncertainty and incompleteness of information; the need for engineering judgment, knowledge of the particular structure, experience of the behaviour of dams in general and a background of general engineering knowledge in order to interpret the data.

\footnotetext{
(C) 1994 IEEE. Personal use of this material is permitted. Permission from IEEE must be obtained for all other uses, in any current or future media, including reprinting/republishing this material for advertising or promotional purposes, creating new collective works, for resale or redistribution to servers or lists, or reuse of any copyrighted component of this work in other works.
} 
Marco Lazzari, Paolo Salvaneschi, "Improved Monitoring and Surveillance through Integration of

Artificial Intelligence and Information Management Systems", Proceedings of the Tenth IEEE

Conference on Artificial Intelligence for Applications (CAIA '94), San Antonio, Texas, 1994

AI concepts and technologies can assist engineers in safety management $[1,2]$ by providing additional components to the existing information system such as real time interpretation systems linked to the data acquisition units and intelligent data bases supporting the off-line management of information and interpretation.

The paper reports the results of the work done in this field during the last four years at the software technologies unit of ISMES. The paper is organised as below:

Chapter 1. describes the pre-existing information system comprised of automatic monitoring systems, telemetry and data bases with associated tools.

Chapter 2. identifies the user requirements driving the evolution of the information system and explains how AI concepts and technologies may contribute.

Chapter 3 illustrates in some detail the functions, the architecture and the AI techniques of the two systems added to the preexisting information system.

Chapter 4. discusses the issue of integration of the AI components and the pre-existing system and presents the technology we developed to support the integration process.

Chapter 5. describes the implementation status of the project which delivered components operational from mid-1992, the user acceptance and some detail about the development effort. Finally the scope of the project and the applicability to other fields are discussed.

\section{The pre-existing information system}

A basic requirement of managing dam safety is the monitoring of the structure in order to collect data which are then interpreted so as to understand the state of the dam. In Italy, this is done through a two level organisational structure: a first level identifies and manages possible alarm conditions and, if necessary, calls the second level; the second level manages the available information concerning the dam and evaluates the safety of the structure on a periodical basis or when requested by the first level.

To provide for the requirements stated above, the pre-existing information system developed by ISMES was comprised of the following subsystems: a real time automatic monitoring system, a telemetry system and a central data base with associated processing and representation functions.

The monitoring system (called INDACO) collects data such as displacements of the dam, basin level, seepage losses, uplift pressures and tests them against thresholds or values predicted by theoretical models (on-line checks). The resulting warnings and associated data are presented to the people working at the first level of the organisation (e.g. the warden of the dam).

The data collected by the monitoring system are sent through a telemetry system to a central computer and are loaded, together with manually collected data, into a data base (called MIDAS), which allows the subsequent analysis and interpretation of the behaviour of the structure (off-line check).

MIDAS has been operational since 1985 and manages the data of 200 dams in different countries (MIDAS has been sold to different organisations in Italy and abroad). MIDAS is running in UNIX and VMS environments. INDACO, a PC-based system, has been installed at 35 sites for dam monitoring.

\section{User requirements and $\mathrm{Al}$ contribution}

The limits of the above described technology and the user requirements for improvement may be classified as belonging to two different levels: local level (management of warnings) and central level (periodical safety evaluation or deep analysis on demand).

At local level, monitoring systems currently available allow the carrying out of two kinds of checks on the values gathered by each instrument:

- comparison of the measured quantity and of its rate of change with pre-set threshold values;

- comparison of the measured quantity 
Marco Lazzari, Paolo Salvaneschi, "Improved Monitoring and Surveillance through Integration of

Artificial Intelligence and Information Management Systems", Proceedings of the Tenth IEEE

Conference on Artificial Intelligence for Applications (CAIA '94), San Antonio, Texas, 1994

with the corresponding value computed

by a reference model.

Therefore, these checks neither deal with more than one instrument at a time, nor with more than one reading at a time for each instrument. In addition, any behaviour (either of the structure, or of the instruments) which is not consistent with the reference model generates a warning message. Because of the limited interpretation skills of the people on-site, false alarms cannot be identified and therefore require expert attention.

At this level, AI may contribute in collecting the expert knowledge related to data interpretation and delivering it through a system linked with the existing monitoring system. The system can filter and classify the anomalies by using different types of knowledge (e.g. geometrical and physical relationships). It can take into account the whole set of measurements and warnings to identify the state of the dam and to explain it. This allows a part of the expert interpretation to be performed on-line, and therefore to reduce the requests for expert intervention and to increase the level of the safety of the dam.

At the central level, the existing system contains quantitative data coming from the monitoring systems. The safety evaluation requires the availability of additional types of information (qualitative data coming from visual inspections, results of experimental tests, design drawings, old interpretations) and different types of knowledge (empirical relationships, numerical models, qualitative models), related to different areas of expertise (structural behaviour, hydrology, geology).

As a consequence, there is a need for a cooperative supporting system, able to help people in managing the complexity of the evaluation. AI can be helpful through providing new ways to model the behaviours of the physical systems (e.g. a qualitative causal net of possible physical processes). This modelling approach is a useful way to integrate different types of knowledge (qualitative and quantitative data and models) providing a global scenario to understand the physical system behaviour. Moreover AI may be helpful in collecting and formalising different types of knowledge from different experts for data interpretation and evaluation of the dam state.

\section{MISTRAL and DAMSAFE}

To improve the capabilities of the information system in accordance with the above stated requirements, we developed two systems using AI concepts and technologies.

The first system, called MISTRAL, operates in real time and is linked to the existing monitoring system. It provides a global interpretation and explanation of the dam state and evaluates it against a desired state.

The second system, called DAMSAFE, may be interpreted as the evolution of the MIDAS data base. It integrates the MIDAS system (data base, graphical and computational tools) and adds new types of information (results of visual inspections and tests, design drawings) It provides additional models of the dam system (a causal net of possible processes occurring in the dam and near environment) and tools to support the interpretation of data and the evaluation of possible scenarios using the causal net.

Below we describe in some detail the functions, the structure and the AI concepts and technologies related to MISTRAL and DAMSAFE. More details may be found in $[3,4$, 5].

\subsection{MISTRAL}

MISTRAL is comprised of the following modules:

푤 communication module: manages the data communication from the monitoring system to MISTRAL;

or evaluation module: identifies the state of the structure;

[D] explanation module: generates a natural-language explanation of the deductions carried out by the evaluation module;

品 man/machine interface: allows the user to access the results of the computation;

(.) database management module: 
Marco Lazzari, Paolo Salvaneschi, "Improved Monitoring and Surveillance through Integration of Artificial Intelligence and Information Management Systems", Proceedings of the Tenth IEEE

Conference on Artificial Intelligence for Applications (CAIA '94), San Antonio, Texas, 1994

manages an internal database of

measurements and evaluations.

The communication module calls the monitoring system and receives the data gathered during the last acquisition (normal real-time procedure) or collected while MISTRAL was, for some reason, not active.

The evaluator and the explainer process these data and store their results in the internal database. The evaluation module executes different types of checks on groups of measurements:

1. checks on sections: readings from instruments within the same section of the dam are composed (e.g. vertical blocks);

2. evaluation of physical phenomena: readings from instruments which measure the same quantity are examined (e.g. displacements, under pressures);

3. evaluation of physical processes: readings from instruments which allow the identification of ongoing processes are examined (e.g. rotation of the dam body, filtering of water through dam body); in this case, the instruments can belong to different sections and measure different quantities.

The result of the checks is the identification of the current state of the structure. This is done through the use of several kinds of representations for codifying the relationships between the data and the states:

- numerical functions: empirical formulas define relations based on the alarm state of single instruments and on their reliability and significance;

- production rules: starting from the values of some attributes of the system, rules can be fired, which set other values as well as the activation state of currently ongoing physical processes;

- constraint based techniques: constraints among variables define possible states of the system; process identification is driven by the satisfaction of those constraints.
From the trace of execution, using knowledge about the behaviour of the dam and the instruments, the explanation module generates natural language messages. They describe the current state of the structure and the deductions of the system. The explanation is built step by step as follows:

- identification of key points of the evaluation process;

- explanation of the key points using texts linked to them and additional knowledge (e.g. knowledge about methods used by the monitoring system to compute the expected value of some quantities);

- display of messages and highlighting of causal links between physical phenomena identified by the evaluator.

The user can access the results of the processing through a window-based interface. The interface draws on the screen graphical representations of the objects which have been assessed (instruments, sections) and displays them using a colour scale based on the state of the object (figure 1). Interactors are available to get more refined information about the dam state, by focusing on interesting details. Through the interface the user can activate functions (e.g. print screen) and access the internal data bases.

MISTRAL provides the users with a static data base of test cases, and a dynamic database collecting all the data related to the control system (measurements, evaluations, explanations). It is possible to select a situation from the data base and show on the screen its graphic representation and explanations.

MISTRAL was developed on a personal computer; the evaluation and explanation modules are written in Prolog, while the remaining modules are implemented with Visual Basic.

\subsection{DAMSAFE}

DAMSAFE is a system in which different types of information (design records, photographs, design drawings, test and monitoring data, qualitative assessments of condition) concerning a dam and different types 
Marco Lazzari, Paolo Salvaneschi, "Improved Monitoring and Surveillance through Integration of

Artificial Intelligence and Information Management Systems", Proceedings of the Tenth IEEE

Conference on Artificial Intelligence for Applications (CAIA '94), San Antonio, Texas, 1994

of models of the dam system (numerical structural models, data models, normative models for behaviour) can be united to assist the engineer in carrying out the procedures of dam safety management (figure 2). The system provides a platform in which the state of the dam system can be represented and then tested against a variety of normative models. The system is intended as a co-operative management tool.

The system developed so far enables hazard audits to be carried out on descriptions of the state and behaviour of the dam coming from monitoring. The structure of the system is based on three main entities contained within an integration environment:

1. models of the physical world

These models can be divided into:

- those which describe the present state of the physical world;

- those which describe desirable states of the physical world;

- those which describe undesirable states of the physical world.

These models are constructed using object-oriented modelling techniques.

2. models of human reasoning (reasoning agents)

These are models of reasoning about the problem domain, including identification of data features or mapping of data states into dam states.

3. communication mechanisms

The communication mechanisms take the form of interfacing software components, which enable the user to co-operate with the system through an object-oriented man/machine interface. The whole system can be used in two different ways:

- as a diagnostic tool: there is a sequence of operations of the reasoning agents that allows the translation of data into dam states;

- as a knowledge integrator: the system assists in the management of safety by facilitating the integration of information about the dam. Drawings, maps and pictures of the dam form part of the information base. Databases of past measurements of the dam can be integrated with the reasoning and modelling system described above. The system functions as an integration tool for different types of knowledge about the dam system, such as theory, regulations and expert knowledge. In such a way the system can be seen as a virtual expert, that reflects the

knowledge of many different experts (civil engineers, hydrologists, geologists,...) interviewed during the knowledge elicitation phase of the development process of the system.

The structure of DAMSAFE is based on the object-oriented metaphor. The different types of knowledge represented within the system are integrated using a hierarchical model describing, through objects and attributes, the main components of the system.

The hierarchical structure is comprised of two physical world models and three reasoning agents. The models make up the problem domain: a model called data world represents all the relevant concepts related to data received from monitoring, while the another model called dam world contains all the relevant concepts related to the physical world of the dam. The reasoning agents act on the physical world models, and contain the knowledge required to reason about the concepts of these models. They perform a variety of tasks, the most important being that of relating the concepts in the data world to those in the dam system world.

Each model represents a view of the physical world, while each reasoning agent represents a function of the data interpretation process performed by dam safety managers.

3.2.1. Data world and dam world: The data world contains several objects; each object represents the data related to a single instrument of the monitoring system. These data are attributes of the object; they can be time series of instrument readings, as well as details of the type of variable represented. Features such as 
Marco Lazzari, Paolo Salvaneschi, "Improved Monitoring and Surveillance through Integration of Artificial Intelligence and Information Management Systems", Proceedings of the Tenth IEEE

Conference on Artificial Intelligence for Applications (CAIA '94), San Antonio, Texas, 1994

peaks, trends, steps and plateaux, identified in different types of time series are recorded in this model.

Each object has methods, which allow the user to access the knowledge linked to the object. In such a way one can read the values of the attributes of the object, or show a time series on the screen. It is also possible to assign values to attributes; this allows the user to act directly on the data world, by-passing the filtering of the reasoning agents.

The dam world contains a model of the physical world of the dam and its environment, concepts describing the possible states of this world and a set of concepts modelling the possible behaviours of the dam and its environment. The physical dam model describes the dam as a hierarchy of objects. Five objects are currently represented within the system: dam body, foundation, curtain wall, basin, and foundation drains. These objects have attributes which, taken as a set, describe the state of the system. The attributes can be quantitative (e.g. reservoir level), qualitative (e.g. concrete quality), or complex (e.g. under pressure profile).

3.2.2. Causal net of processes: The model of the behaviours of the dam system is a set of processes connected in a causal network. The causal network models how behaviours of the dam and its environment can interlink in a causal way resulting in scenarios as one process leads to another. The full net includes ninety different processes describing possible dam behaviours (e.g. translation, clogging of drains in dam body, chemical degradation of foundation). This network has been derived from published case studies of dam failures and accidents, and from discussions with experts in the field of dam design and safety. The conditions under which one process can lead to another have been included. Each of these processes has been documented along with descriptions of how evidence of these processes might be manifested in the monitoring data and also in reports from visual inspections.

The network can be used in different ways:

- as a data base: each process has attributes, which describe the process itself (e.g. start time, rate of change, activation state). The system provides methods for accessing such attributes, in order to show the user their values;

- as a control panel of the system: each process is represented on the screen by a box, that is highlighted whenever the system infers that the process is active. Therefore the highlighted boxes give the user an immediate synthetic report on the current state of the dam. Besides the activation state, other attributes (reversibility, speed) are represented by coloured areas of the box linked to the process representation on the screen;

- as an inference tool: the causal links can be used by automatic reasoners for building paths of events to be used for simulating the future evolution of the system state or identifying the possible causes of an active process;

- as a knowledge base: each process is linked in a hypertextual way to its written documentation, that describes the process and its connections to other entities (processes and objects).

Therefore the theoretical foundations of the system itself can be easily accessed through the user interface.

3.2.3. Reasoning agents: Three reasoning agents have been developed. The first one (extractor) operates solely on the data world to manipulate the data and extract features from the data sets of importance. It uses the graphical interface to show to the user a time series plot and to interactively identify a set of features of the plot which are considered relevant to dam safety. Possible features are trends, spikes, steps and plateaux. They are defined by qualitative and quantitative attributes (e.g. spike length, start time) and stored within the data world. These attributes can also be accessed and manipulated through methods of the data world.

The second reasoning agent (mapper) performs the task of interpretation identifying both the 
Marco Lazzari, Paolo Salvaneschi, "Improved Monitoring and Surveillance through Integration of

Artificial Intelligence and Information Management Systems", Proceedings of the Tenth IEEE

Conference on Artificial Intelligence for Applications (CAIA '94), San Antonio, Texas, 1994

possible behaviours of the dam in terms of a set of processes in the causal net, and the values of various attributes of the dam, based on evidence in the data. This task is performed by firing rules defined by experts, which link data values to dam states. These links are defined by using a formal language designed with the aim of allowing non-programmers to easily write and read rules. The rules are translated into $\mathrm{C}++$ code, compiled and then executed by the mapper. A rule is fired if a precondition on the values of some data world attributes is verified; in this case, the state of some dam world process is declared active and some dam world attributes receive a value. The set of active processes linked in a causal chain are highlighted by the system and describe a scenario that demonstrates the evolution of the dam behaviour.

The third reasoning agent (enforcer) acts on the dam world to extend the implications of the state identified by reasoning agent 2 , over the model of the dam and its environment thus highlighting possible causal chains.

Once a model has been built of the state of the dam system in terms of a set of active processes and a set of attributes, this state can be tested against normative models to make judgements about the safety of the dam.

\section{The integration of $\mathrm{Al}$ components}

Software systems traditionally used in engineering have integrated different types of physical world models (e.g. numerical models or data bases) and communication mechanisms (e.g. graphical and textual representation or man/machine interfaces).

In this kind of system, models of human decisions have been implemented, but normally they are neither clearly identified nor modelled as reasoning agents but are embedded in the code.

AI concepts and technologies provide new approaches to modelling, such as qualitative causal models of physical systems, and the ability to write reasoning agents, for instance including associations used for data interpretation. This extends the capabilities of traditional software technologies allowing the development of new and more complex software systems.

From a technical point of view the integration with the pre-existing systems was very simple for MISTRAL. On the contrary DAMSAFE uses a specific technology that we developed. The basic idea is that a software system designed and implemented around the integration concept is made of two layers.

A first layer contains the software components to be integrated. For example in this layer we can find data bases, procedures, sets of rules, qualitative causal models, images or programs producing graphical representations.

The second layer is a model based integrator. This is a software tool whose aim is to provide an environment where specific components can be encapsulated. The concept providing the structure of the integrator is that the integration is made through one or more software models. The models, implemented using an object oriented technology, are an abstraction of the existing system of software components.

The integrator is composed of the following layers:

- abstractions (software models);

- communication mechanisms between models and users (man/machine interface);

- communication mechanisms between models and the system of software components.

Following this approach we implemented an environment called MI using $\mathrm{C}++$ and the InterViews toolkit of the X-Window System under UNIX.

MI contains three layers of objects' classes. The first layer implements the communication channel through the screen; the second implements one or more software models, while the third provides the communication mechanisms with the existing software components. More details may be found in $[6$, 7]. 
Marco Lazzari, Paolo Salvaneschi, "Improved Monitoring and Surveillance through Integration of Artificial Intelligence and Information Management Systems", Proceedings of the Tenth IEEE

\section{Status of the implementation}

A version of MISTRAL has been installed since October 1992 on a PC linked to the INDACO monitoring system of the Ridracoli dam (an arch-gravity dam, length about $500 \mathrm{~m}$., max. height about $100 \mathrm{~m}$.) and is currently in use. Another version has been completed in 1993 for the Fiastrone dam. The system at Ridracoli dam obtained very rapid acceptance by the user. It reduced the effort required for the management of warnings and, most importantly, improved the quality of the safety management procedures.

DAMSAFE is now integrated with the preexisting off-line system MIDAS and is entering the operational phase at ISMES.

MISTRAL and DAMSAFE required a global effort of 9 man/years. DAMSAFE consists of about 100,000 lines of C++; MISTRAL includes 2,000 lines of Prolog and 15,000 lines of Visual Basic. The development of the system required a team of people with different professional backgrounds (civil engineers, experts in dams safety, software engineers and programmers).

The applicability of the approach is much wider than the safety management of dams. It is related to the application of monitoring systems to structural and environmental problems. For example in 1993 we developed a version of MISTRAL devoted to the real time interpretation of the structural behaviour of the Cathedral of Pavia.

\section{Acknowledgements}

The development of the systems above described was supported by ENEL/DSR/CRIS (Research Centre on Hydraulics and Structures of the Italian Electrical Company).

\section{References}

1. B. M. Franck, T. Krauthammer, Preliminary safety and risk assessment for existing hydraulic structures - an expert system approach, Report ST-87-05, Univ. of Minnesota, Dept of Civil and Mineral Eng., Inst. of Technology, 1987.

2. D. Grime, T. Phillips, M. Waage, "The Kelly system: on-line expertise", Hydro Review, August 1988.

3. J.B. Comerford, P. Salvaneschi, M. Lazzari, P. Bonaldi, G. Ruggeri, M. Fanelli, G. Giuseppetti, G. Mazzà, "The role of AI technology in management of dam safety: the DAMSAFE system", Dam Engineering, 3(4), 1992.

4. J.B. Comerford, M. Lazzari, G. Ruggeri, P. Salvaneschi, M. Fanelli, G. Giuseppetti, G. Mazzà, "Causal models and knowledge integration in system monitoring", 2nd Colloquium on Knowledge-Based Systems in Civil Engineering, Beijing, China, 1993, Int. Assoc. for Bridge and Structural Engineering (IABSE).

5. M. Lazzari and P. Salvaneschi, "MISTRAL - An expert system for the management of warnings from automatic monitoring systems of dams", ICADERS - 2nd Specialist Seminar, Ljubljana, Slovenia, 1993.

6. P. Salvaneschi, "The use of integration tools in software maintenance", IEEE Conf. on Software Maintenance, Sorrento, Italy, 1991.

7. J.B. Comerford, M. Lazzari, D. Pina, P. Salvaneschi, "An AI approach to the integration of engineering knowledge: water resources case studies", 7th Int. Conf. on Applications of Artificial Intelligence in Engineering (AIENG 92), Waterloo, Ontario, 1992.

Fig 1 - The MISTRAL system

Fig 2 - The DAMSAFE system

\footnotetext{
(C) 1994 IEEE. Personal use of this material is permitted. Permission from IEEE must be obtained for all other uses, in any current or future media, including reprinting/republishing this material for advertising or promotional purposes, creating new collective works, for resale or redistribution to servers or lists, or reuse of any copyrighted component of this work in other works.
} 